C-A/AP/\#185

February 2005

\title{
Estimate of $\mathrm{Cu}-\mathrm{Cu}$ Run Experimental Background at Phobos
}

S.Y. Zhang

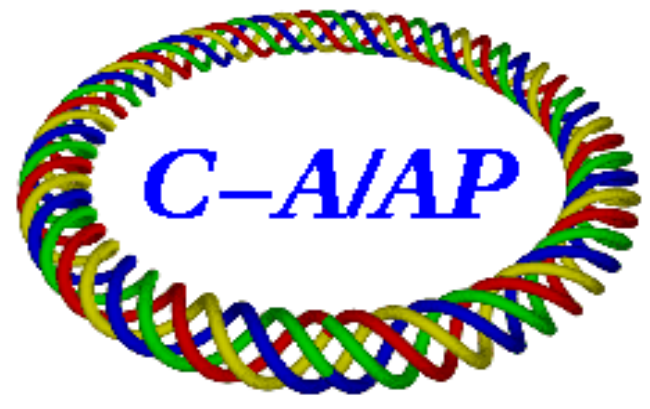

Collider-Accelerator Department Brookhaven National Laboratory Upton, NY 11973 


\title{
Estimate of Cu-Cu Run Experimental Background at Phobos
}

\author{
S.Y. Zhang
}

\section{Abstract}

One of the intensity limits in RHIC $2004 \mathrm{Au}-\mathrm{Au}$ run was the Phobos background, which was caused by the pressure rise at the beam rebucketing. In $2005 \mathrm{Cu}-\mathrm{Cu}$ run, this background problem may not be as serious as that in $\mathrm{Au}-\mathrm{Au}$ run. The background of beam-gas in $\mathrm{Cu}-\mathrm{Cu}$ run due to pressure rise is about $1 / 3$ of that in $\mathrm{Au}-\mathrm{Au}$ run, given same pressure rise. The background due to beam-beam nuclear collision is also about 1/3 of that in $\mathrm{Au}-\mathrm{Au}$ run. As the result, relatively higher beam intensities with higher pressure rises might be allowed in copper run without interfering with the experimental data taking at the Phobos.

\section{Introduction}

The pressure rise of Phobos at the beam rebucketing was one of main beam intensity limits in $2004 \mathrm{Au}-\mathrm{Au}$ run. The Phobos background was clearly correlated with this pressure rise $[1,2]$, and usually data was not taken until a sudden drop of this pressure rise in a period of 20 minutes to 2 hours. In later run, 45 bunch mode was used to substitute the 56 bunch mode for the concerns of this pressure rise.

In $2005 \mathrm{Cu}-\mathrm{Cu}$ run, the higher bunch intensity with a factor of 2 of the gold is available at the injector, 28 bunch mode is adopted in the beginning of the run, which gives rise to about the same total beam intensity as the last $\mathrm{Au}-\mathrm{Au}$ run. The pressure rises at the beam transition and rebucketing are comparable to that observed in $\mathrm{Au}-\mathrm{Au}$ run, however, the ratio of background vs. ZDC coincidence rate looks much smaller than that in $\mathrm{Au}-\mathrm{Au}$ run. The 
ZDC coincidence rate can be predicted from the copper ions nuclear collision. The background is mainly from two sources as far as the Phobos is concerned. One is the single Coulomb dissociation rate from the beam-beam nuclear collision, and another is the beam-gas collision. The former is somewhat better understood, whereas the later is not [3].

In this note, we show that the background due to single Coulomb dissociation from the beam-beam nuclear collision is lower in $\mathrm{Cu}-\mathrm{Cu}$ run than in $\mathrm{Au}-\mathrm{Au}$ run, given comparable $\mathrm{ZDC}$ rate. This is illustrated from both the theory and the observed data.

We also show that the background due to beam-gas collision is lower in $\mathrm{Cu}-\mathrm{Cu}$ run than in $\mathrm{Au}-\mathrm{Au}$ run, for given comparable pressure and beam intensity. This is illustrated mainly from the observed data.

As the result, for same beam intensity, $\mathrm{Cu}-\mathrm{Cu}$ run has lower background/ZDC ratio. Therefore, higher beam intensity than the $\mathrm{Au}-\mathrm{Au}$ run can be allowed without affecting experimental data taking.

\section{Parameters}

\subsection{ZDC coincidence rate}

The experimental ZDC coincidence rates are mainly from the beam ions nuclear collision and the mutual Coulomb dissociation. For the former the cross section can be calculated using 'billiard ball' model, which gives rise to $\sigma_{A u}=6.1 b\left(1 b=10^{-28} m^{2}\right)$ for gold, and $\sigma_{C u}=2.9 b$ for copper. Usually a mutual Coulomb dissociation cross section of $4 b$ is taken for $\mathrm{Au}$ [3]. Since this cross section is scaled approximately as $Z^{6}$ [4], it can be neglected for copper. For instance, the mutual Coulomb dissociation cross section of $\mathrm{Ca}$ is believed to be only $0.001 b[4]$.

Therefore, the total cross section responsible for $\mathrm{Au}-\mathrm{Au}$ coincidence rate is

$\sigma_{A u Z D C}=6.1 b+4 b=10.1 b$ and for $\mathrm{Cu}-\mathrm{Cu}$ coincident rate it is $\sigma_{C u Z D C}=2.9$

$b$. In the calculation, we use $\sigma_{A u Z D C}=11 b$ as suggested in [4].

\subsection{Beam-beam singles}

The beam-beam singles rate can be calculated from the single Coulomb dissociation cross section, which for gold is usually taken as $\sigma_{\text {AusigBB }}=92 b$ $[3,4]$. The single Coulomb dissociation can be scales approximately as $Z^{3}$ [4], 
therefore, we take the single Coulomb dissociation cross section for copper as $\sigma_{C u s i g B B}=4.7 \mathrm{~b}$.

\subsection{Beam-gas singles}

For beam-gas nuclear collision, if one uses the cross section calculated using 'billiard ball' model, then the rate is much smaller than the observations. Therefore, it was speculated in [3] that a $Z^{2}$ effect enhanced beam-gas ionization may playing a role in the beam-gas singles. In this note, cross sections of beam-gas collision for gold and copper are obtained by fitting into the experiment observations.

\section{Observations}

Two fills in $\mathrm{Au}-\mathrm{Au}$ run and $\mathrm{Cu}-\mathrm{Cu}$ run are used for illustration. These are 4832, with 45 bunch mode, in Au-Au run 2004, and 5853, with 48 bunch mode, of $\mathrm{Cu}-\mathrm{Cu}$ run 2005. Both had Phobos rebucketing pressure rise, which lasted a while, then suddenly dropped. This way the beam-gas and beambeam induced background can be roughly identified.

In Figure 1, the beam intensity, the Phobos pressure rise at G9 and G10, and the Phobos ZDC and background of 4832 are shown.

In Figure 2, the beam intensity, the Phobos pressure rise at G9 and G10, and the Phobos ZDC and background of 5853 are shown.

In Figure 3, the observed ZDC coincidence rates of 4832 and 5853 are shown in blue lines. The calculated one in red lines are also shown. The cross sections discussed in last section are used. The calculation methods are the one shown in [3]. Detailed parameters are in the figure caption, some discussion is also shown there.

It can be noticed that the $\mathrm{ZDC}$ rate in 4832 was contaminated by the high pressure before 64 minutes, and it is also there for 5853 before 26 minutes, but it is small.

In Figure 4, the observed background of 4832 is shown in blue, and the simulated one is in red line. The background includes both beam-beam collision due to the single Coulomb dissociation $\left(\sigma_{\text {AusigBB }}=92 b\right.$ for gold $)$, and the beam-gas collision. For the beam-gas created singles, a cross section of $\sigma_{\text {AusigBG }}=100 \mathrm{~b}$ is used for better fitting. Other parameters are listed in the figure caption. 
In Figure 5, the observed background of 5853 is shown in blue, and the simulated is in red line. For copper beam-beam collision created singles, the cross section $\sigma_{C u s i g B B}=4.7 b$ is used. For the beam-gas created singles, a cross section of $\sigma_{C u s i g B G}=33 b$ for copper is used for better fitting. Other parameters are listed in the figure caption.

\section{Results and Discussion}

To fit the observed background of Phobos in $\mathrm{Au}-\mathrm{Au}$ run and $\mathrm{Cu}-\mathrm{Cu}$ run, the beam-gas collision cross section can be obtained. This cross section is scaled approximately as $Z$, leading to the result that the copper-gas collision cross section is about $1 / 3$ of that for gold-gas collisions. Therefore, for given pressure rise, the background due to the beam-gas in copper run is lower than the one in gold run.

Also, without the contribution of vacuum pressure rise, the ratio of background vs the ZDC for gold is

$$
\frac{\sigma_{A u s i g B B}+\sigma_{A u Z D C}}{\sigma_{A u Z D C}}=\frac{92 b+11 b}{11 b}=9.4
$$

and for copper it is

$$
\frac{\sigma_{C u s i g B B}+\sigma_{C u Z D C}}{\sigma_{C u Z D C}}=\frac{4.7 b+2.9 b}{2.9 b}=2.6
$$

This is approximately agreeable with the observations in past $\mathrm{Au}-\mathrm{Au}$ run and the current $\mathrm{Cu}-\mathrm{Cu}$ run. For example, in fills of 4829 to 4832 for $\mathrm{Au}-\mathrm{Au}$ run, and the fills 5905-5907 for $\mathrm{Cu}-\mathrm{Cu}$ run. Therefore, the background due to the beam-beam collision is also smaller in copper run than in gold run.

Some discussions are as follows.

1. Previous experiences have shown that the ratio of background/ZDC below 5 is acceptable, 10 is marginal, and above 20 it is not acceptable. This has been learned in both d-Au run in 2003, and $\mathrm{Au}-\mathrm{Au}$ run in 2004 .

2. The beam-gas collision induced background is clearly harmful. On the other hand, the beam-beam singles is inherent, and it is not very clear how much it is affecting the data taking. 
3. Considering the beam-gas collision induced background, a factor of 3 high pressure rise than that in $\mathrm{Au}-\mathrm{Au}$ run can be tolerated at the Phobos. If total background/ZDC ratio is more important, then even higher pressure rise may be tolerated.

4. The mechanism of beam-gas collision created background is not understood, however, the observed one shown in Phobos for $\mathrm{Cu}-\mathrm{Cu}$ and $\mathrm{Au}-\mathrm{Au}$ runs, with the scaling of $Z$, is interesting and needs interpretation.

\section{References}

1. A. Drees, U. Iriso, and W. Fischer, 'Correlation of Pressure Rise and Experimental Background at RHIC in Run04', in ECLOUD'04, Napa, California, Apr. 2004.

2. S.Y. Zhang et. al, 'RHIC Pressure Rise', EPAC 2004, Lucerne, Switzerland, July 2004.

3. S.Y. Zhang, "Experiment Background in RHIC Deuteron-Gold Run "C-AD/AP 107, Sep. 2003.

4. A.J. Baltz, C. Chasman, and S.N. White, NIM A417, p.1, 1998. 

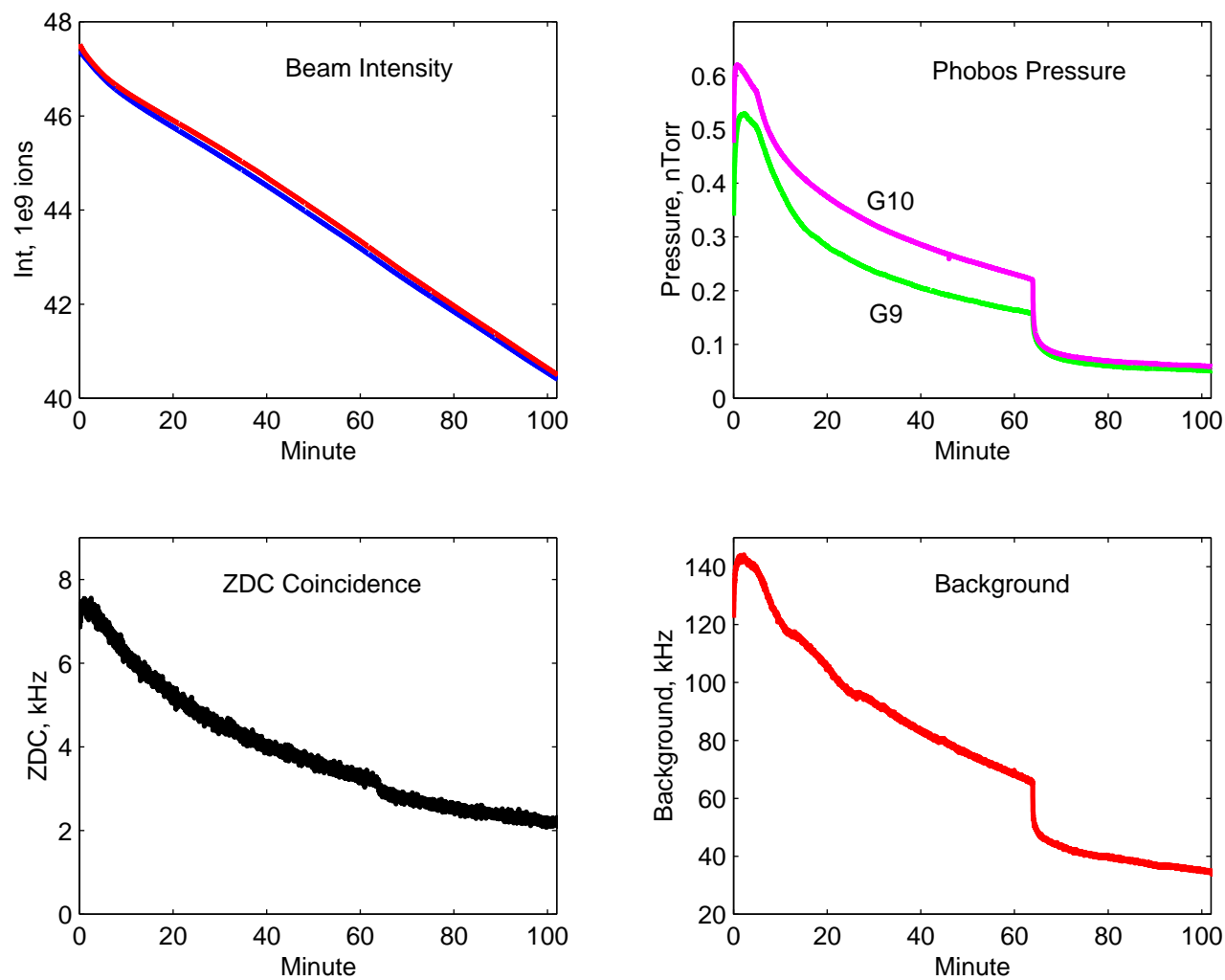

Figure 1: Fill 4832 in Run 2004. Au-Au run, with 45 bunch mode. The beam intensity, the Phobos pressure rise at G9 and G10, and the Phobos ZDC and background are shown. 

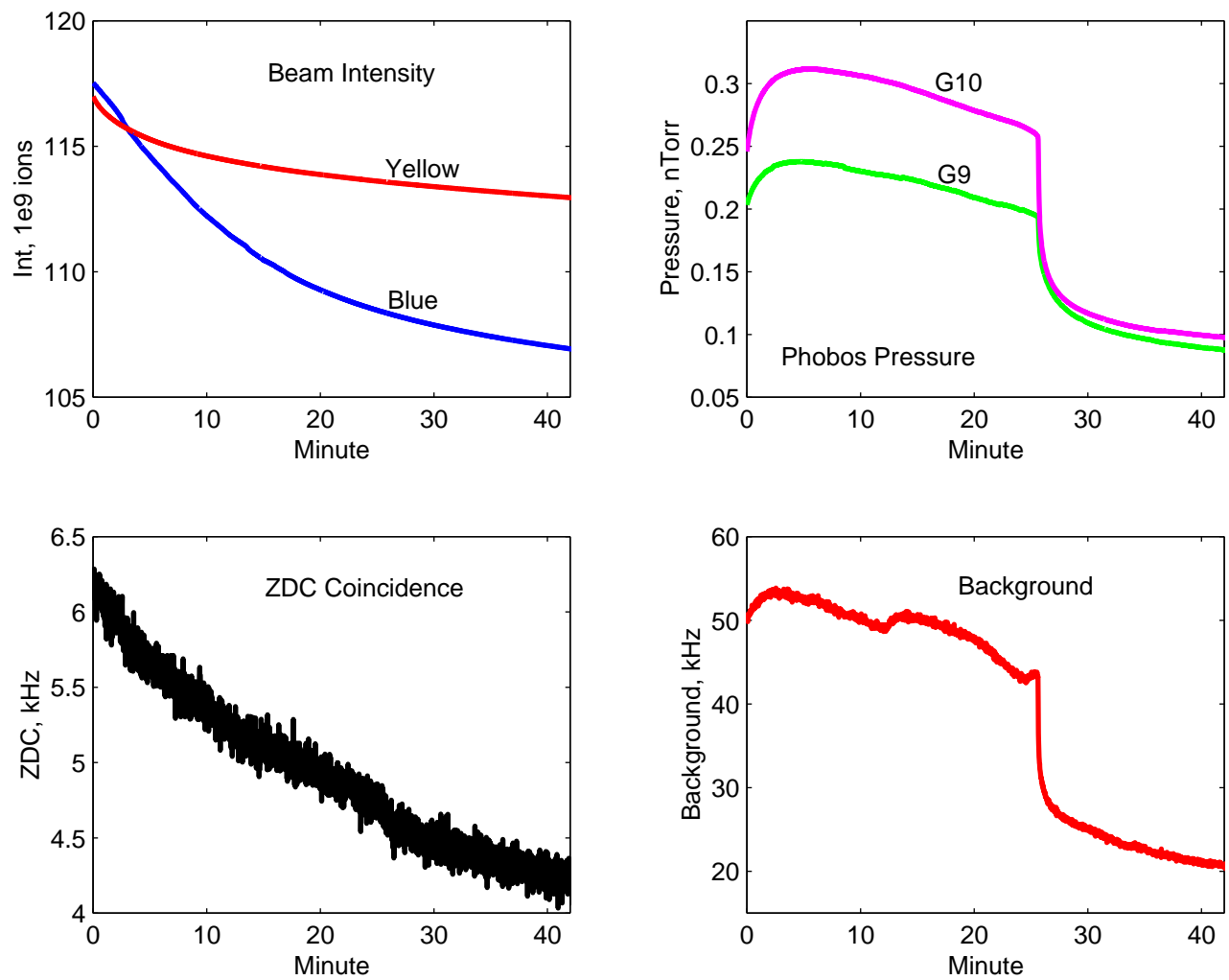

Figure 2: Fill 5853 in Run 2005. Cu-Cu run, with 48 bunch mode. The beam intensity, the Phobos pressure rise at G9 and G10, and the Phobos ZDC and background are shown. 

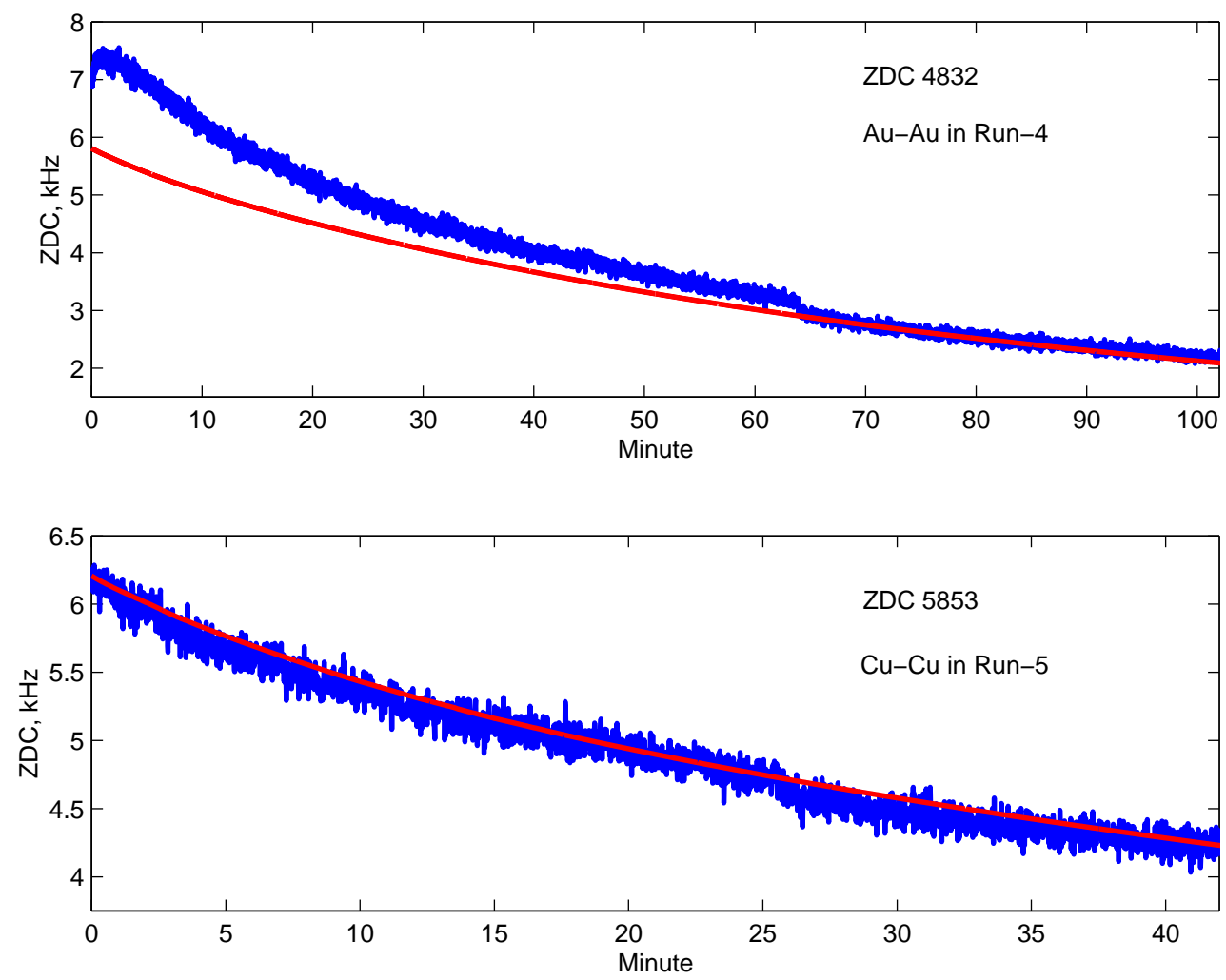

Figure 3: Blue lines are the observed ZDC coincidence of 4832 and 5853. The red lines are from the calculation. The parameters used for 4832 of $\mathrm{Au}-\mathrm{Au}$ collision are: $\beta^{*}=3 \mathrm{~m}$ at the Phobos, ZDC coincidence cross section $\sigma_{A u Z D C}=11 b$ in [4] is used, with 45 bunches the bunch intensity is calculated from the DCCT beam intensity shown in Figure 1, beam collision length is $1.5 \mathrm{~m}$ ( $5 \mathrm{~ns}$ ), with $\beta \gamma=106$, the beam normalized emittance is started from $8.5 \pi \mu m$, and it is doubled in 100 minutes. Since the DCCT rather than the bunch intensity is used, this emittance growth is actually exaggerated for better fiting, but this is not important for this note. The parameters used for 5853 of $\mathrm{Cu}-\mathrm{Cu}$ collision are: $\sigma_{C u Z D C}=2.9 \mathrm{~b}$. Other parameters are the same as that for 4832 , except that the beam normalized emittance is started from $12 \pi \mu m$, and it is doubled in 145 minutes. 


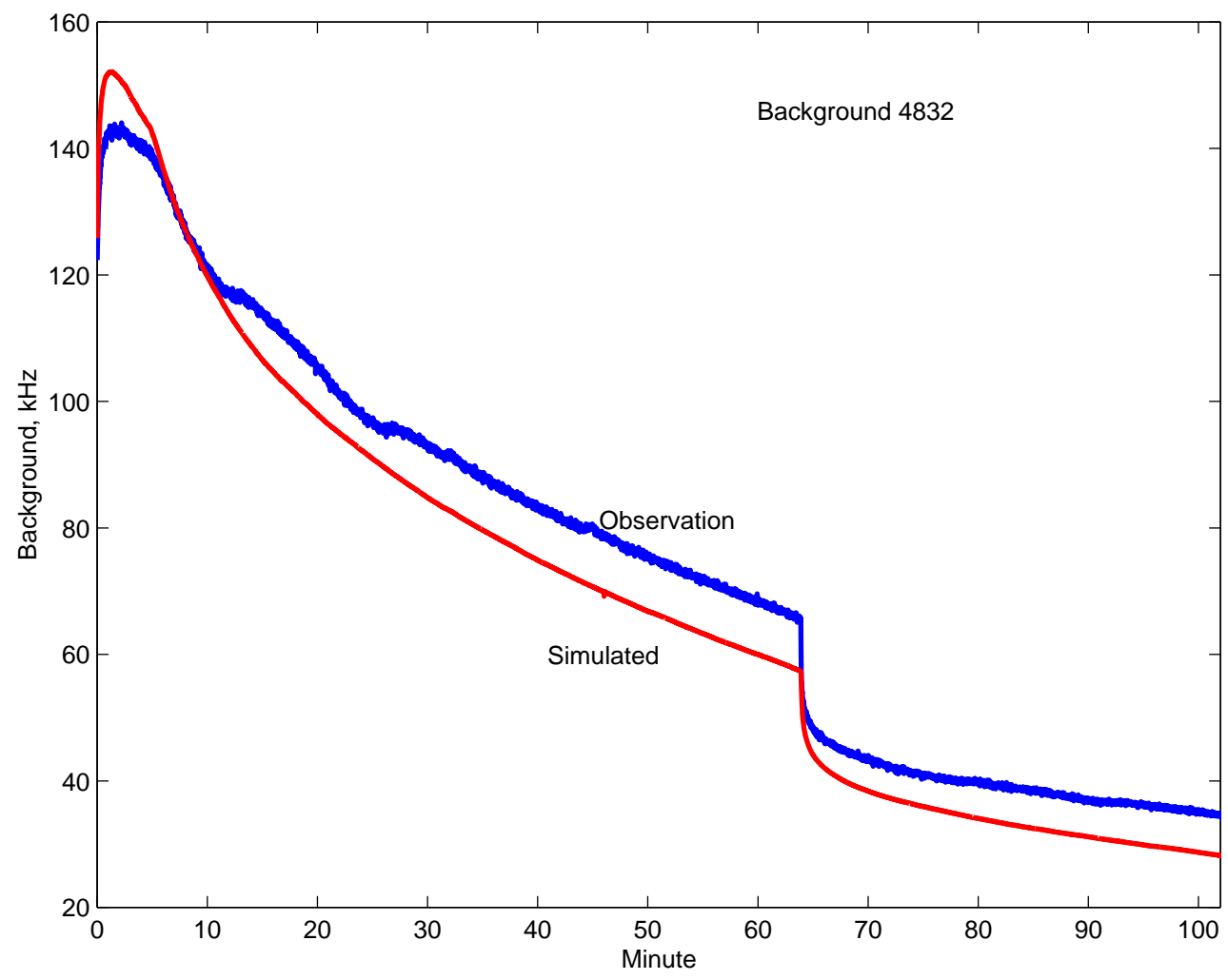

Figure 4: Blue line is the observed background of 4832, and the red line is the simulated. Note that the background consists of beam-gas and beambeam collisions. The sudden drop of the background at 64 minutes is due to the pressure drop, as shown in Figure 1. The parameters in the background calculation are: for beam-beam collision, the single Coulomb dissociation cross section of $\sigma_{\text {AusigBB }}=92 b$ is used. For the beam-gas created singles, a cross section of $\sigma_{A u s i g B G}=100 b$ is used for better fitting. The vacuum conditions of the ones in [3] are used, which assumes that $14 \mathrm{~m}$ long beamgas collision length with the real pressure rise averaged by a factor of 10 compared with the G9 and G10 vacuum gauges, which are located at DX magnet and far away from the center. 


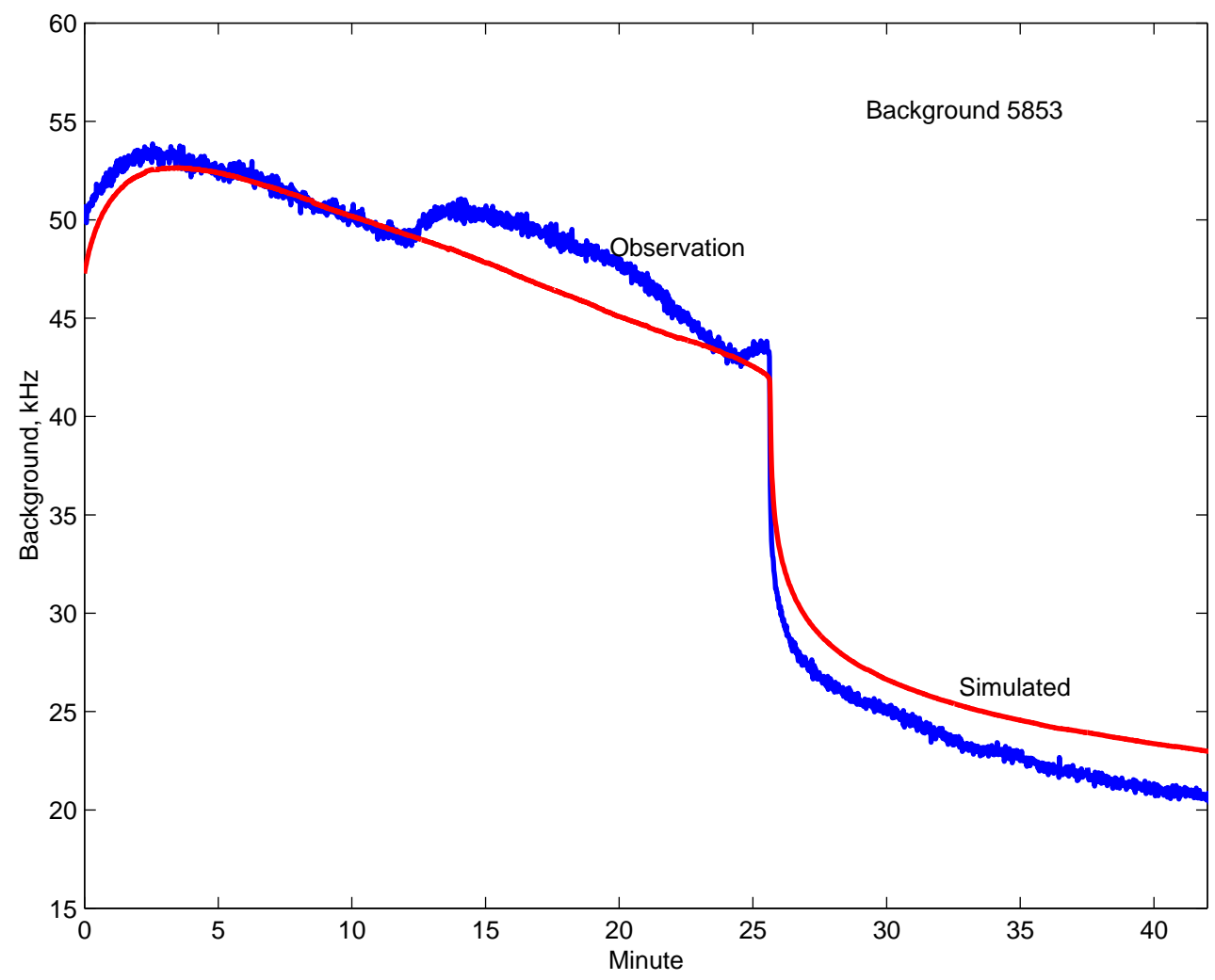

Figure 5: Blue line is the observed background of 5853, and the red line is the simulated. The sudden drop at 26 minutes is due to the pressure drop, as shown in Figure 2. The parameters in the background calculation are: for beam-beam collision, the single Coulomb dissociation cross section of $\sigma_{\text {CusigBB }}=4.7 \mathrm{~b}$ for copper is used. For beam-gas collision, the vacuum conditions of the same as that in Figure 4 is used, except that the beam-gas cross section of $\sigma_{\text {CusigBG }}=33 b$ for copper is used. 